\title{
18-Month Performance Assessment of Gemini TF 16 PET/CT System in a High-Volume Department
}

\author{
Ashish Kumar Jha, Sneha Mithun, Abhijith Mohan Singh, Nilendu C. Purandare, Sneha Shah, Archi Agrawal, \\ and Venkatesh Rangarajan
}

Department of Nuclear Medicine and Molecular Imaging, Tata Memorial Hospital, Parel, Mumbai, India

\begin{abstract}
Acceptance testing is a set of quality control tests performed to verify various manufacturer-specified parameters before a newly installed PET/CT system can be accepted for clinical use. A new PET/CT system, Gemini TF 16, installed in our department in September 2012 has a PET component capable of time-of-flight imaging using lutetium-yttrium-oxyorthosilicate crystals and operates in 3-dimensional mode. Our aim was to evaluate the system before acceptance and observe the consistency of its performance during high-volume work for 18 mo after installation (we perform an average of 30 PET/CT scans daily). Methods: We performed NEMA (National Electrical Manufacturers Association) NU-2 2007 acceptance testing on the Gemini TF 16; continuously evaluated its gain calibration, timing resolution, and energy resolution during the subsequent $18 \mathrm{mo}$; and analyzed the results. Results: The system passed the acceptance testing and showed few fluctuations in energy and timing resolutions during the observation period. Conclusion: The Gemini TF 16 whole-body PET/CT system performed excellently during the 18-mo study period despite the high volume of work.
\end{abstract}

Key Words: PET; PET/CT; time-of-flight; fully 3-dimensional whole-body imaging; LSO; LYSO; NEMA QC

J Nucl Med Technol 2016; 44:36-41

DOI: $10.2967 /$ jnmt.115.168492

\section{A} ssessment of the performance of any newly installed medical device is important to ensure the quality of clinical outcomes. Likewise, daily quality control testing ensures the consistency and reproducibility of performance. The final diagnostic outcome of a medical imaging device depends largely on the quality of the images produced (1). Therefore, their reliability should initially be judged under nonclinical conditions through a battery of quality assurance tests.

Agencies such as the National Electrical Manufacturers Association (NEMA), the International Atomic Energy Agency,

Received Oct. 19, 2015; revision accepted Jan. 13, 2016.

For correspondence or reprints contact: Ashish Kumar Jha, Department of Nuclear Medicine and Molecular Imaging, Tata Memorial Hospital, Parel, Mumbai 400012, India.

E-mail: ashish.kumar.jha.77@gmail.com

Published online Feb. 4, 2016.

COPYRIGHT (c) 2016 by the Society of Nuclear Medicine and Molecular Imaging, Inc. and the medical physicist associations of various countries prescribe guidelines to assess the performance of medical equipment after initial installation and after any major repair before it is accepted for clinical use. These agencies also prescribe various quality assurance tests to check the performance of medical equipment at regular intervals during its life cycle. In the United States, NEMA publishes quality assurance guidelines for a variety of medical equipment, such as the NEMA NU-2 guidelines for PET systems $(2,3)$.

In September 2012, our institution installed a Gemini TF 16 whole-body PET/CT system (Philips), which combines a high-performance PET scanner with a 16-slice Brilliance CT scanner. The Gemini TF 16 acquires and stores data in list mode, is capable of time-of-flight imaging, and acquires data in 3-dimensional mode only, as there are no septa rings between the PET detector rings $(4,5)$. The system's lutetiumyttrium-oxyorthosilicate (LYSO) scintillation detectors have characteristics similar to lutetium oxyorthosilicate (LSO) and an excellent timing resolution, with dead time of 470 ps. Lutetium and yttrium are at a 9:1 ratio, and the presence of a trace amount of intrinsic radioactive ${ }^{177} \mathrm{Lu}$, a positron emitter, contributes a significant number of random coincidence events at low counting rates (6).

After passing all performance tests prescribed in NEMA NU-2 2007, the system was licensed by the Atomic Energy Regulatory Board of India before being put into clinical use. As part of our quality control program, the system is tested every morning and is not used clinically unless all parameters are within the prescribed limits.

\section{MATERIALS AND METHODS}

\section{PET/CT System}

The PET component of the Gemini TF 16 consists of 28,336 LYSO crystals arranged in a $23 \times 44$ array on each of 28 flatmodule detectors, which are formed into 44 complete rings (644 crystals per ring). Each detector is $4 \times 4 \times 22 \mathrm{~mm}$, and each ring has a diameter of $906 \mathrm{~mm}$ and an axial length of $180 \mathrm{~mm}$. The patient bore is $716 \mathrm{~mm}$ in diameter, with active transverse and axial fields of view of 675 and $180 \mathrm{~mm}$, respectively. PET and CT are performed sequentially, with the PET acquisition being in stepand-shoot mode and keeping a 50\% overlap for the axial field of view. The system works in time-of-flight mode and acquires data in list mode $(7,8)$. Data are reconstructed using a highly advanced iterative algorithm, and a CT attenuation map is used to correct the PET images. 


\section{Phantoms}

The daily quality control PET phantom is used for daily timing calibration and quality assessment; the SUV phantom, for SUV calibration; the PET/CT alignment phantom, for alignment of the CT images with the PET images; and the spatial resolution phantom (a triangular phantom made of plastic sheeting), for placement of the point source at the NEMA NU-2 2007-specified positions within the field of view.

The scatter phantom is a solid cylindric phantom used to measure scatter, random events, dead time, count loss, and noise equivalent counting rate (NECR). The outer diameter of the cylinder is $203 \mathrm{~mm}$, and the length is $700 \pm 3 \mathrm{~mm}$. There is a $6.4-\mathrm{mm}$ hole across the length at a $45-\mathrm{mm}$ offset, which is used to place the line source in the phantom. The inner and outer diameters and the length of the line source are 3.2, 5, and $800 \mathrm{~mm}$, respectively (9).

The sensitivity phantom contains 700-mm-long internally stacked concentric aluminum tubes. The respective inside and outside diameters are 3.9 and $6.4 \mathrm{~mm}$ for the first tube, 7.0 and $9.5 \mathrm{~mm}$ for the second, 10.2 and $12.7 \mathrm{~mm}$ for the third, 13.4 and $15.9 \mathrm{~mm}$ for the fourth, and 16.6 and $19.1 \mathrm{~mm}$ for the fifth. The innermost fillable polyethylene tube has an inside diameter of $1 \mathrm{~mm}$ and an outside diameter of $3 \mathrm{~mm} \mathrm{(10).}$

The image-quality body PET phantom is the NEMA NU-2 2007 standard, which has an interior depth of $180 \mathrm{~mm}$ and 6 spheric inserts with inner diameters of $10,13,17,22,28$, and $37 \mathrm{~mm}$. The distance from the sphere plane to the inside wall is $70 \mathrm{~mm}$. The volume of the D-shaped cylinder is $9.7 \mathrm{~L}$. In the middle of the phantom is a cylindric polystyrene ball-filled lung insert having an outer diameter of $51 \mathrm{~mm}$ and a length of $180 \mathrm{~mm}(11)$.

\section{Quality Control Tests}

Various acceptance tests were performed and the results were compared with the reference values provided by the manufacturer. Non-NEMA tests were performed using the manufacturer's protocol. The NEMA NU-2 2007 tests were performed for spatial resolution, scatter fraction, sensitivity, counting rate loss, random coincidence rate, NECR, and image quality.

Normalization and SUV calibration tests were performed after successful installation of the system followed by an SUV verification test, after which all other tests were performed.

PET/CT Coregistration. The PET and CT images were coregistered using $6{ }^{22} \mathrm{Na}$ tablet sources placed on specific sites on the PET/CT coregistration phantom. Serial CT and PET images were acquired, and software was used to reveal any errors in coregistration in the $x, y$, and $z$ coordinates.

Energy Calibration. Using a ${ }^{22} \mathrm{Na}$ point source, an energy spectrum was generated for each crystal, its peak was individually calculated, and the peaks of all crystals were normalized to a common value using energy correction tables generated for the individual crystal elements. The system energy resolution is calculated by averaging over all crystals after energy correction for $511 \mathrm{keV}$. The default energy window is $440-665 \mathrm{keV}$.

Timing Calibration. The Gemini TF 16 is designed to work in time-of-flight mode, which requires accurate timing information for each annihilation event. Timing calibration was performed using a ${ }^{22} \mathrm{Na}$ point source.

Energy Resolution. Energy resolution is the ability of an imaging system to distinguish between two energy peaks closely associated with each other. The energy resolution of the PET system is represented by the full width at half maximum and the full width at tenth maximum of the energy spectrum.
Spatial Resolution. The spatial resolution of PET systems varies across the scanning field of view in the axial and transaxial directions. Therefore, per NEMA NU-2 2007, spatial resolution was calculated in the axial and transaxial directions at 6 points $(2,3)$.

Sensitivity. The sensitivity of a PET system is the rate of detection of true coincidence events per unit of radioactivity concentration. In order to compare the sensitivity of different systems, measured sensitivity values need to be free of confounding effects such as attenuation, scatter, and counting rate distortions $(2,3)$.

Scatters, Count Losses, and Randoms. Scatters and randoms are PET imaging phenomena that introduce invalid events, hence affecting both image quality and the quantitative accuracy of a system.

The scatter fraction is the ratio of the scatter coincidence count to the total coincidence count when random coincidence events are negligible, as occurs at low counting rates. PET systems consisting of scintillation crystals with intrinsic radioactivity (such as LSO and LYSO) have a substantially high intrinsic random coincidence count, which can be corrected by using delayed imaging $(3,11,12)$.

Random coincidence counts are the counts that emerge from two independent events that are detected in the same coincidence window. There are mainly two types of random events, intrinsic and extrinsic, that are detected in the LYSO crystal-based detector. Intrinsic randoms are due to intrinsic radioactivity present in the detector, and extrinsic randoms are due to an increasing counting rate. Randoms introduce unwanted counts to the image and hence are corrected to achieve better image quality.

Counting rate performance is the ability of a PET system to accurately measure high-radioactivity sources as well as lowradioactivity sources. Because clinical studies frequently are performed with levels of radioactivity for which count losses due to system dead time are considerable, and the rate of randoms increases with the total single-event counting rate, the measurement of count losses and randoms at various levels of activity is of the utmost importance.

NECR is an important parameter to consider before performing any test that requires injection of a high amount of radioisotope. The NECR of a PET system is the counting rate performance of the system as a function of radioactivity concentration. Peak NECRs and corresponding radioactivity concentrations are used to determine the optimal radioactivity to administer to individual patients. NECR was calculated using the NEMA NU-2 2007 formula (3).

Accuracy Corrections for Count Losses and Randoms. The data acquired to calculate scatter fraction were also used to measure net error in counting rate after correction for dead-time losses and randoms. Reconstructed data from a $65-\mathrm{cm}$ scatter phantom were used to calculate accuracy corrections for count losses and randoms using standard NEMA NU-2 2007 parameters.

Image Quality and Accuracy of Attenuation and Scatter Correction. Image quality and accuracy were tested using the NEMA NU-2 2007 image-quality and scatter phantoms. The image-quality phantom allows simulation of whole-body imaging with hot and cold lesions. The NEMA NU-2 2007 formula was used to calculate image contrast, background variability, and the accuracy of attenuation and scatter correction. NEMA NU-2 2007 image-quality testing was performed twice during our study period: at acceptance testing and 12 mo later.

\section{Eighteen-Month Observation Period}

Every day, our staff works from 8:00 AM to 7:00 PM and performs about 30 whole-body PET/CT procedures on the single Gemini TF 16 system. Hence, the system was observed for 18 mo after its installation to assess its stability. Measurements of key parameters such as photomultiplier tube (PMT) gain calibration error, energy 
TABLE 1

Comparison of Image Quality at Acceptance Testing and 12 Months Later

\begin{tabular}{|c|c|c|c|c|c|c|}
\hline \multirow[b]{2}{*}{ Parameter } & \multicolumn{6}{|c|}{ Insert diameter } \\
\hline & $10 \mathrm{~mm}$ & $13 \mathrm{~mm}$ & $17 \mathrm{~mm}$ & $22 \mathrm{~mm}$ & $28 \mathrm{~mm}$ & $37 \mathrm{~mm}$ \\
\hline \multicolumn{7}{|l|}{ Contrast recovery } \\
\hline At acceptance testing & $34.70 \%$ & $51.43 \%$ & $60.83 \%$ & $72.13 \%$ & 73.88 & $77.65 \%$ \\
\hline 12 mo later & $36.30 \%$ & $50.62 \%$ & $60.13 \%$ & $70.69 \%$ & 72.12 & $76.92 \%$ \\
\hline Difference & $+1.6 \%$ & $-0.81 \%$ & $-0.60 \%$ & $-1.44 \%$ & $-1.76 \%$ & $-0.73 \%$ \\
\hline \multicolumn{7}{|l|}{ Background variability } \\
\hline At acceptance testing & $8.65 \%$ & $7.89 \%$ & $7.05 \%$ & $6.15 \%$ & $5.46 \%$ & $5.04 \%$ \\
\hline 12 mo later & $8.96 \%$ & $8.01 \%$ & $6.85 \%$ & $6.65 \%$ & $5.77 \%$ & $5.32 \%$ \\
\hline Difference & $+0.31 \%$ & +0.12 & $-0.20 \%$ & $+0.50 \%$ & $+0.31 \%$ & $+0.28 \%$ \\
\hline
\end{tabular}

Scatter correction using filtered backprojection reconstruction was applied.

TABLE 2

Comparison of Lesion and Background SUVs for Image-Quality Phantom at Acceptance Testing and 12 Months Later

\begin{tabular}{|c|c|c|c|c|c|c|c|}
\hline \multirow[b]{3}{*}{ SUV site } & \multicolumn{6}{|c|}{ SUV... } & \multirow[b]{3}{*}{ Error ${ }^{*}$} \\
\hline & \multicolumn{3}{|c|}{ at acceptance testing } & \multicolumn{3}{|c|}{12 mo later } & \\
\hline & Maximum & Minimum & Average & Maximum & Minimum & Average & \\
\hline Lesion 1 (22 mm) & 4.95 & 2.1 & 3.04 & 4.7 & 1.98 & 2.97 & $5.05 \%$ \\
\hline Lesion 2 (17 mm) & 4.65 & 1.98 & 2.84 & 4.53 & 1.93 & 2.75 & $2.58 \%$ \\
\hline Lesion $3(13 \mathrm{~mm})$ & 3.91 & 1.63 & 2.2 & 3.73 & 1.58 & 2.12 & $4.60 \%$ \\
\hline Lesion $4(10 \mathrm{~mm})$ & 2.35 & 0.96 & 1.44 & 2.33 & 1.08 & 1.50 & $0.85 \%$ \\
\hline Background & 1.36 & 0.98 & 1.20 & 1.34 & 1.03 & 1.15 & $1.47 \%$ \\
\hline
\end{tabular}

${ }^{\star}$ Error in SUV $\max$ estimation in second study compared with first study.

TABLE 3

Daily Quality Control Results During the 18-Month Observation Period

\begin{tabular}{lccrr}
\hline Parameter & $\begin{array}{c}\text { Iterations required for } \\
\text { gain calibration }\end{array}$ & Gain calibration error (\%) & $\begin{array}{c}\text { Average energy } \\
\text { resolution (\%) }\end{array}$ & Timing resolution (ps) \\
\hline Average & 10.493 & 0.238 & 11.664 & 547.075 \\
SD & 0.812 & 0.026 & 0.147 & 1.772 \\
\hline
\end{tabular}

resolution, and timing resolution were gathered once a week from daily quality control records, analyzed, plotted, and compared with the measurements at acceptance testing. Mean and SD over the 18-mo observation period were also determined. In addition, the temperature of the room housing the PET/CT system was monitored and recorded during the observation period, and the types of PET/CT procedures performed were noted. NEMA NU-2 2007 imagequality testing was repeated 12 mo after installation, and the data were compared with those at installation. Raw data from the imagequality tests were reconstructed using iterative technique. The SUVs of each spheric insert and its background were measured and compared.

\section{RESULTS}

\section{Installation and 12-Month Tests}

The Gemini TF 16 was installed successfully and passed the SUV verification test. The accuracy of PET/CT image registration as tested using a $512 \times 512$ matrix was well within the permissible limit. Timing resolution was 542.3 ps; energy resolution was 11.2\%; and the respective tangential, radial, and axial resolutions were $4.84,5.06$, and $4.73 \mathrm{~mm}$ near the center and 4.89, 5.03, and $5.19 \mathrm{~mm}$ at $10 \mathrm{~cm}$ off-center.

Absolute sensitivity as measured with a 70-cm-long line source using the NEMA NU-2 2007 sensitivity phantom was $7,901 \mathrm{cps} / \mathrm{MBq}$, and scatter fraction as measured with a 70-cm-long line source placed in the 20-cm-diameter NEMA NU-2 2007 scatter phantom was $24.712 \%$. For the same line source cylinder, peak NECR was $112,499 \mathrm{kcps}$ at a 0.015 $\mathrm{MBq} / \mathrm{mL}$ activity concentration, peak coincidence counting rate was $263,975 \mathrm{kcps}$ at $0.01652 \mathrm{MBq} / \mathrm{mL}$, and $50 \%$ deadtime loss in the coincidence count occurred at $0.028 \mathrm{MBq} / \mathrm{mL}$.

Testing using the NEMA NU-2 2007 image-quality and scatter phantoms showed that image quality was within the specified limit and did not differ much between the time of acceptance and 12 mo later (Table 1). The SUVs of the spheric inserts and their background at acceptance testing were similar to those obtained 12 mo later (Table 2). 


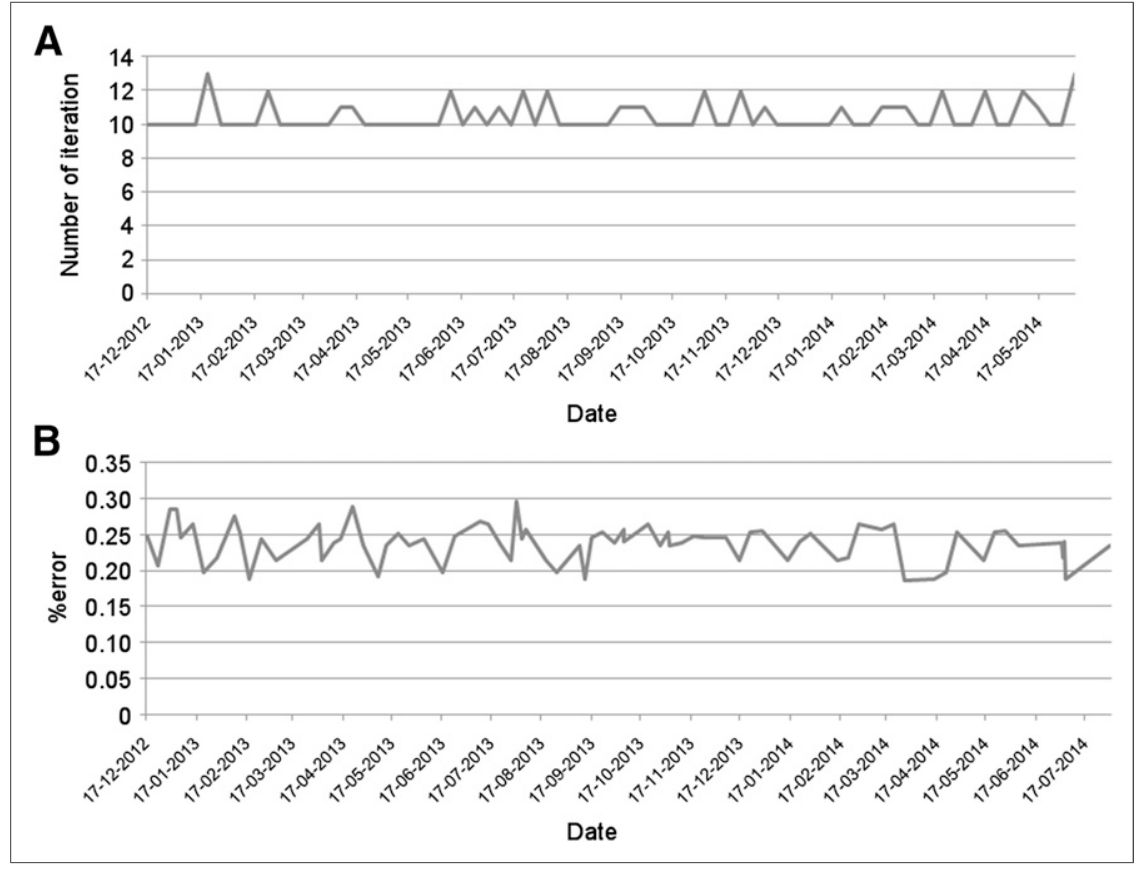

FIGURE 1. (A) Variations in number of iterations required for gain calibration during observation period. (B) Variations in average error in gain calibration during observation period.

\section{Eighteen-Month Observation Period}

The total number of PET/CT scans performed over the 18 mo was 8,473 , with a daily average of $28 \pm 4$. There was little fluctuation in the daily quality control parameters (Table 3).

The total number of iterations required for gain calibration is shown in Figure 1, and the error in gain calibration averaged $0.238 \% \pm 0.026 \%$. Energy resolution averaged $11.664 \% \pm$ $0.147 \%$ (Fig. 2), versus $11.2 \%$ at acceptance testing. Timing resolution averaged $547,045 \pm 1,772 \mathrm{ps}$ (Fig. 3), versus 542.3 ps at acceptance testing, and remained within the manufacturer's pass limit on all occasions (passing with notice on 3 occasions).
Although the temperature of the room housing the PET/ CT system was monitored, the data were not stored properly. On 3 occasions the temperature increased beyond $30^{\circ} \mathrm{C}$, with the maximum recorded temperature being $36^{\circ} \mathrm{C}$. The maximum temperature fluctuation in a day was approximately $5^{\circ} \mathrm{C}-6^{\circ} \mathrm{C}$.

\section{DISCUSSION}

PET/CT has become established as an important diagnostic modality for staging, restaging, and follow-up in oncologic imaging. Two important characteristics of PET systemsimage resolution (to detect small lesions) and imaging speed (to increase patient throughput)—are determined mainly by

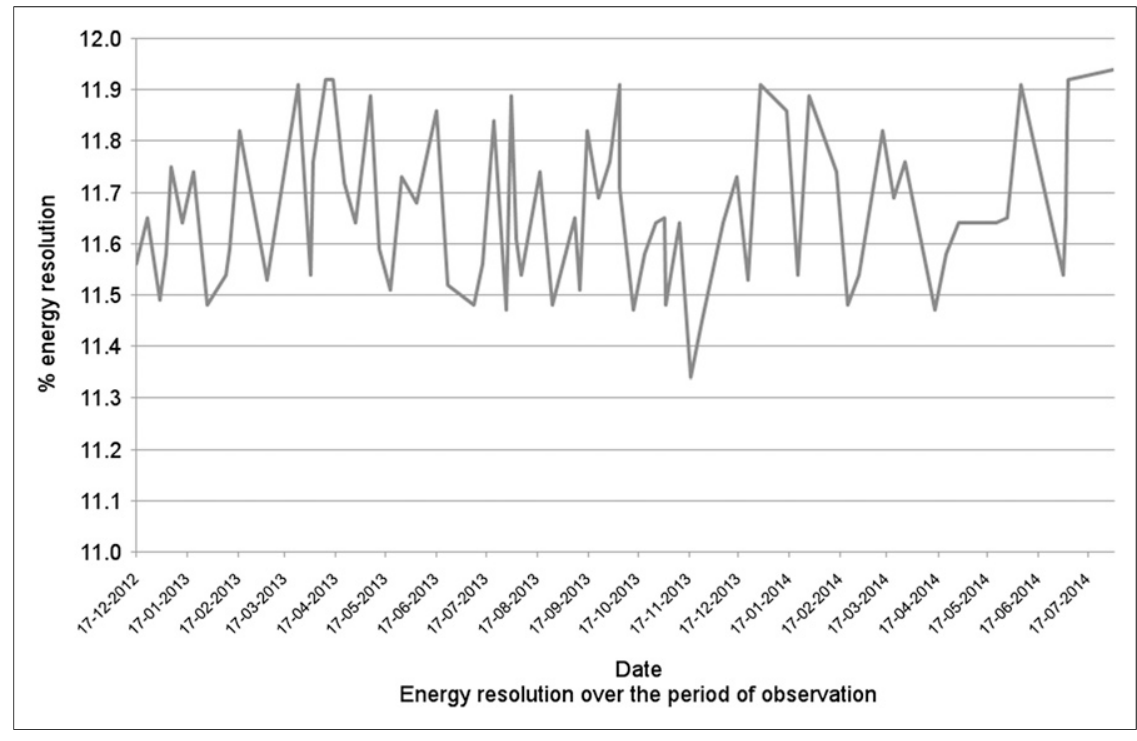

FIGURE 2. Variations in system energy resolution during observation period. 
FIGURE 3. Variations in system timing resolution during observation period.

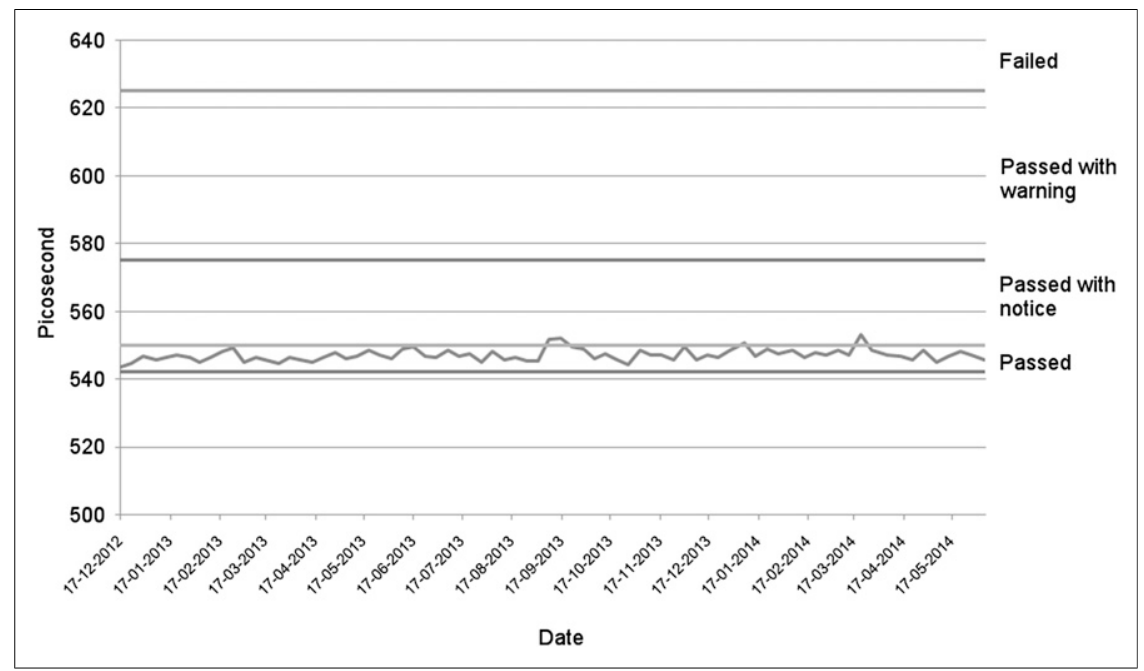

how detector crystals identify events emerging from within the patient's body (7). The advent of LSO and ceriumdoped LYSO crystals, which have an excellent timing resolution of about $49 \mathrm{~ns}$, enabled the introduction of timeof-flight technology to clinical PET, with the first system being the Gemini TF PET/CT developed by Philips in 2005 $(7,12,13)$.

The scintillation detector of the Gemini TF 16 uses LYSO crystals, whose low dead time, high stopping power, and high photon yield allow high sensitivity, high resolution, and time-of-flight imaging with excellent timing resolution (5). LSO- and LYSO-based PET detectors contain the natural radioactivity of ${ }^{177} \mathrm{Lu}$, which emits intrinsic randoms during the acquisition $(3,14,15)$. The presence of this intrinsic activity even at very low counting rates contributes randoms to the true counts at a rate that cannot be kept below $1 \%$, thus preventing use of NEMA NU-2 2001 testing. Modifications that account for the intrinsic randoms by counting in a delayed window were suggested by Erdi et al. and Watson et al. $(14,15)$. Watson's suggestion was incorporated into NEMA NU-2 2007, which uses delayed-window counting. NEMA NU-2 2007 also suggests that, in PET systems that have LSO or LYSO crystals, the delayed count be subtracted from the prompt count to correct for intrinsic randoms (3).

Scatter is one factor responsible for PET image degradation. The lower-level discriminator setting is important for minimizing scatter in the image $(16,17)$. In the Gemini TF 16, the use of an Anger-logic detector having a uniform spread of light coupled with LYSO crystals has allowed the lower threshold of the energy window to be set as high as $440 \mathrm{keV}$, thereby enabling excellent energy resolution (full width at half maximum, 11\%) at the photopeak and reduced scatter correction during image acquisition $(7,8)$. The ability of time-of-flight imaging to improve image quality was not tested in our study but has qualitatively been perceived as obvious. An im- portant parameter for fusion imaging devices is alignment of the gantry. Gantry misalignment can significantly degrade image coregistration. In our system, PET and CT image coregistration was excellent, with an error of less than $2 \mathrm{~mm}$.

To check the consistency of the system, we compared the various parameters at the time of acceptance testing with those at daily quality control testing. We did not find much change during the 18-mo observation period. Comparisons with acceptance testing showed that the timing and energy resolutions were quite stable. The average error in gain calibration was minimal. The daily quality control reports showed consistency in the electronic and overall stability of the system despite the huge workload.

\section{CONCLUSION}

The performance of the Gemini TF 16 under high-volume conditions is robust. The system not only passed all initial NEMA NU-2 2007 acceptance tests but also, for 18 mo afterward, showed no significant variations on daily quality control tests, all of which it passed.

\section{DISCLOSURE}

No potential conflict of interest relevant to this article was reported.

\section{REFERENCES}

1. Quality control of nuclear medicine instruments 1991. International Atomic Energy Agency website. http://www-pub.iaea.org/MTCD/Publications/PDF/te_602_web. pdf. Accessed January 20, 2016.

2. NEMA Standards Publication NU2-2001: Performance Measurements of Positron Emission Tomographs. Rosslyn, VA: National Electrical Manufacturers Association; 2001.

3. NEMA Standards Publication NU2-2007: Performance Measurements of Positron Emission Tomographs. Rosslyn, VA: National Electrical Manufacturers Association; 2007. 
4. Surti S, Karp JS, Popescu LA, Daube-Witherspoon ME, Werner M. Investigation of time-of-flight benefit for fully 3-D PET. IEEE Trans Med Imaging. 2006; 25:529-538.

5. Griesmer J, Laurence T, Cooke S, Karp J, Perkins A, Kolthammer J. Time-of flight quality control for a new Philips Gemini PET/CT scanner. J Nucl Med. 2006;47(suppl 1):391-392.

6. Pepin CM, Bérard P, Perrot A-L, et al. Properties of LYSO and recent LSO scintillators for phoswich PET detectors. IEEE Trans Nucl Sci. 2004;51:789795.

7. Surti S, Kuhn A, Werner ME, Perkins AE, Kolthammer J, Karp JS. Performance of Philips Gemini TF PET/CT scanner with special consideration for its time-offlight imaging capabilities. J Nucl Med. 2007;48:471-480.

8. Gregory R, Partridge M, Flower MA. Performance evaluation of the Philips “Gemini” PET/CT system. IEEE Trans Nucl Sci. 2006;53:93-101.

9. PET cylinder phantom acc NEMA NU2-2007. PTW website. http://www.ptw. de/pet_cylinder_phantom_nema.html?\&cId=4259. Accessed January 20, 2016.

10. PET sensitivity phantom acc NEMA NU2-2007. PTW website. http://www.ptw. de/pet_sensitivity_phantom_nema.html?\&cId=4259. Accessed January 20, 2016.
11. PET emission phantom acc NEMA NU2-2007. PTW website. http://www.ptw. de/pet_emission_phantom_nema.html?\&cId=4259. Accessed January 20, 2016.

12. Melcher CL, Schweitzer JS. A promising new scintillator: cerium-doped lutetium oxyorthosilicate. Nucl Instrum Methods. 1992;A314:212-214.

13. Bonificio DAB, Belcari N, Moehrs S, et al. A time efficient optical model for GATE simulation of a LYSO scintillation matrix used in PET applications. IEEE Trans Nucl Sci. 2010;57:2483-2489.

14. Erdi YE, Nehmeh SA, Mulnix T, Humm JL, Watson CC. PET performance measurements for an LSO based combined PET/CT scanner using the NEMA NU 2-2001 standard. J Nucl Med. 2004;45:813-821.

15. Watson CC, Casey ME, Eriksson L, Mulnix T, Adams D, Bendriem B. NEMA NU 2 performance tests for scanners with intrinsic radioactivity. $J$ Nucl Med. 2004; 45:822-826.

16. Ollinger JM. Model-based scatter correction for fully 3D PET. Phys Med Biol. 1996;41:153-176.

17. Bergström M, Eriksson L, Bohm C, Blomqvist G, Litton J. Correction for scattered radiation in a ring detector positron camera by integral transformation of the projections. J Comput Assist Tomogr. 1983;7: $42-50$. 